\title{
Zigbee Cluster Tree Performance Improvement Technique
}

\author{
Rajeshwari Uikey and Sanjeev Sharma \\ School of Information Technology, UTD, RGPV School of Information Technology, UTD, RGPV \\ Bhopal Madhya Pradesh 462033 India Bhopal Madhya Pradesh 462033India
}

\begin{abstract}
Zigee is the communication protocol designed for wireless networks. It has low rates, low complexity, cost and power consumption also for connectivity with portable. Due to increasing demand for mobile services and in the same way mobile devices consume the network services of the service provider.Every network have their own pros and cons with lot of scope for enhancement of network. Zigbee network offers higher performance over different parameters seen the promising structure of network, So to achieve better performance many researchers proposed zigbee cluster tree network. Still there is scope for modification to enhance the zigbee network. To complete this demand and to achieve high performance network Zigbee cluster-tree network, we propose method to investigate their promises and their performance over different performance parameters, and also proposed the required changes.To justify modification for the model, implementation ofmodifications made in the network is performed and comparing performance of theproposed technique with existing Zigbee cluster network tree. Inproposed method work Enhance the algorithm for Zigbee Cluster tree network which is compatible with IEEE802.15.4 standard.
\end{abstract}

\section{Keywords}

Zigbee cluster tree network, implementation, performance study, comparison, changes of model.

\section{INTRODUCTION}

Recent advances in wireless communications and microelectromechanical Technologies have had a strong impact on the development of wireless sensor networks (WSNs) [1]. ZigBee is the only standards-basedwe ask that authors follow some simple guidelines. Due to wired technologies some further investigation wireless technology designed and focused on basic needs of low-cost, low-power wireless sensor and control networks. ZigBee can be easy to implement and less power required to operate, the opportunity for growth into new markets, as well as the innovation workin existing markets, is limitless. The applications supported by ZigBee include home automation schemes, remote control and monitoring systems and healthcare devices too.

ZigBee cluster-tree topology may not be able to provide sufficient bandwidth for the increased traffic load; the additional information may not be delivered successfully. The common drawback of the existing approaches is that they do not address the poor bandwidth utilization problem inZigBee cluster-tree networks, so it is difficult to increase the system throughput.

The research article [2] based on the Zigbee cluster network and an adoptive-parent-based framework for a ZigBee clustertree network proposed by the author. The author of this article is to provide more flexible routing and increase bandwidth utilization without violating the operating principles of the ZigBee cluster-tree protocol. The paper proposed, design and implement an approach and simulate their performance using simulation results. But according to the author of this research article "However, the restricted routing and poor bandwidth utilization in a ZigBee clustertree network cannot be performed after traffic load increasing and also not provided sufficient bandwidth, Due to this the additional information cannot be delivered successfully. Adpative Parent baesd framework for Zigbee cluster treeis proposed in order to increase the bandwidth utilization without incurring any extra message exchange. Under the framework, a throughput maximization problem, called the vertex-constraint maximum flow problem, is formulated, and proposed distributed algorithm is fully compatible with the ZigBee standard. The theoretical analysis proves that the proposed algorithm can provide an optimal solution, and the results of simulation experiments demonstrate a significant performance improvement over the original approach."

This paper is presented as follows. Section 2 an overview of Zigbee, its supports protocol stack and cluster tree network. Section 3 describes the literature which helpful in our work. Section 4 Describe the problem. Section 5 present experimental setup and result comparisons.Section 6 complete evaluation of performance with concluded.

\section{OVERVIEW OF THE ZIGBEE NETWORK}

ZigBee is the only standards-based technology that addresses the unique needs of most remote monitoring and control sensor network applications. The ZigBee standard was developed to address the following needs: Low cost, Secure, Reliable and self healing, Flexible and extendable, Low power 
consumption, Easy and inexpensive to deploy, Global with use of unlicensed radio bands, integrated intelligence for network set-up and message routing.

\subsection{Zigbee Devices}

ZigBee networks include the following device types: Coordinators, Routers and End devices.

Coordinator device working with starts and controls the network. The coordinator stores information about the whole network, which act as the Trust Center part of the network and being the repository for security keys purpose.

Router devices focusing on extending network area coverage, dynamically route around with obstacles, and provide backup routes in case of network congestion or device failure occurrence. They can connect to the coordinator and other routers, and also support child devices.

End Devices also known as child device but child device is not always known as an end device. It can transmit or receive a message, but cannot perform any routing operations. They must be connected to either the coordinator or a router, and do not support child devices.

Combined the characteristics of the IEEE 802.15.4 standard with the maturing ZigBee specification in defining the wireless profiles for low data rate monitoring and control applications [3].Zigbee and 802.15.4 are tightly coupled shows in below Fig.1 protocol stack to provide the consumer standardization for low-power and low-rate wireless communication devices.

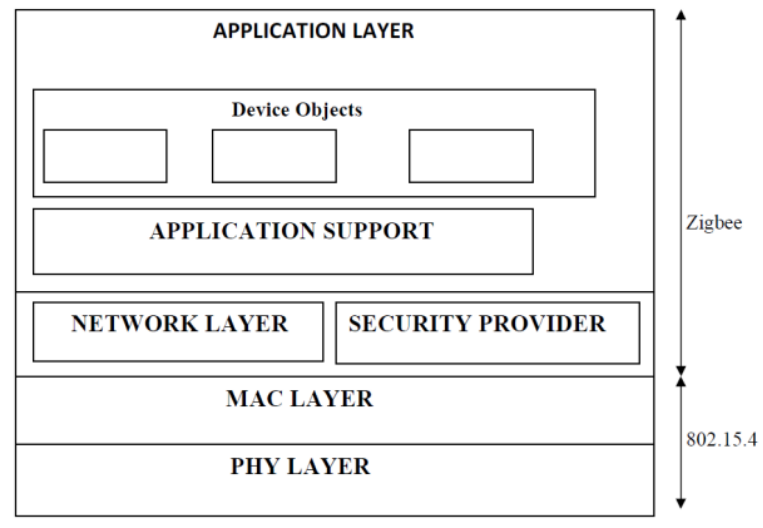

Fig.1 Zigbee protocol Stack

\subsection{Types of Networks}

Zigbee supported topologies are star topology, cluster topology and mesh topology. Cluster tree base topology is effective for WSNs. But it has some restriction in routing poor bandwidth utilization.

In a tree structure, any link failure will suspend data delivery completely and the recovery operation will incur a considerable overhead. The topology also prevents the use of many potential routing paths, which means that a considerable amount of bandwidth cannot beutilized. In a constructedWSN, the information about any area of interest may require further investigation As a result, the sampling rate of the sensor nodes deployed in the area of interest will be increased, and more traffic will be generated suddenly in the network. However, as noability of ZigBee cluster-tree topology to provide sufficient bandwidth in case of Traffic load increased suddenly, the additional information may not be delivered successfully [2].

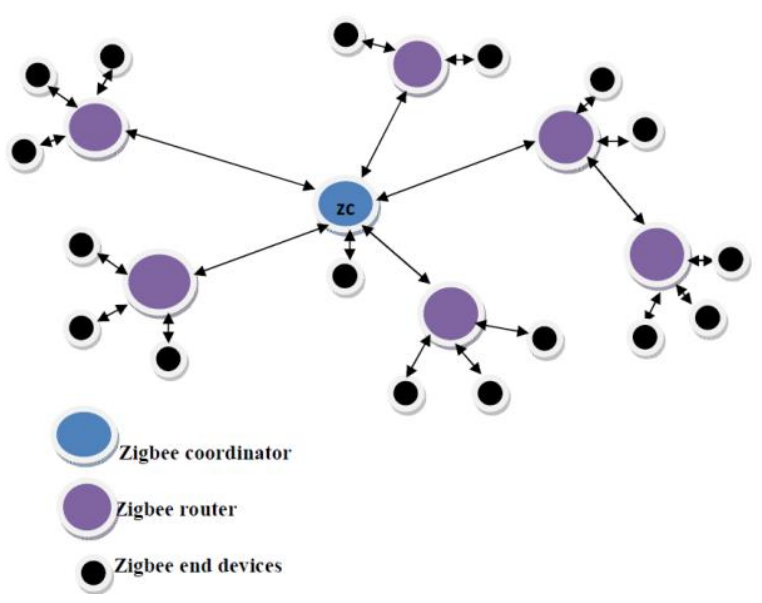

Fig.2 Zigbee/IEEE801.15.4 cluster network and device types

\section{LITRATURE REVIEW}

ZigBee and IEEE 802.15.4 are two standards-based protocols for providing the network infrastructure required for wireless sensor network applications. 802.15.4 defines the physical and MAC layers, and ZigBee defines the network and application layers. Weighted Centroid Localization (WCL) provides a fast and easy algorithm to locate devices in wireless sensor networks. The algorithm is derived from a centroid determination which calculates the position of devices by averaging the coordinates of known reference points .In this positioning algorithm not give desired but presented localization algorithm with a combination of Zigbee offers a lot advantages [4]. Synchronization of multi-hop cluster-tree network problem solved [5]. In which, the current IEEE 802.15.4/Zigbee specifications restrict the synchronization in the beacon-enabled mode (by the generation of periodic beacon frames) to star-basednetworks, while it supports multihop networking using the peer-to-peer mesh topology, but with no synchronization. Even though both specifications mention the possible use of cluster-tree topologies, which combine multi-hop and synchronization features, the description on how to effectively construct such a network topology is missing. This paper tackles this problem, unveils the ambiguities regarding the use of the cluster-tree topology and proposes a synchronization mechanism based on Time Division Beacon Scheduling to construct cluster-tree WSNs.

In [6] we have studied a Multipath Energy Aware AODV (ME-AODV) routing to improve the performance of existing ZigBee routing protocol. ME-AODV divides the ZigBee network into logical clusters. The proposed algorithm 
helps this logical cluster information to reduce the routing overhead in the network. Along with the clustering technique a blend of Multipath routing and Minimum Battery Cost Routing (MBCR) has also cooperated to increase the lifetime of the network by load balancing of energy consumption. Through ME-AODV we get an effort to reduce the number of route discovery and contribute to improving the network performances such as Network Lifetime, Packet Delivery Ratio and Routing Overhead. The simulation also shows that ME-AODV significantly improves mentioned network performance parameters.

For the need oh high reliability in wireless communication through IEEE 802.15.4/Zigbee get meshing capability which is till loose synchronization in the networks. The goal in the networks is also energy efficient but it requiredcycle management in close synchronization. In cluster tree topology beacon scheduling centralized butinmeshing not be defined to overcome [7] a distributed beacon scheduling (DBS) algorithm proposed. In routing algorithm [8] suggest forwarding packet to the neighbor node for reducing routing is explained. It is an efficient term of both time complexity and routing performance, also reduced routing cost. According performance evaluation it reduced more than 30 percent of the routing cost required for regular Zigbee tree routing. Also comparison between Bluetooth, UWB, Zigbee and Wi-Fi is wireless communication is standards. Evolutions on their main features are including the transmission time, power consumption, data coding efficiency and complexity [9]. The routing cost to destination. Zigbee provides routing protocols are tree routing and table-driven.

Image Transmission also included in Zigbee networks .Quality of the image had compared with wireless transmission between 100 JPEG and 100 JPEG-2000 images over the networks that support multi-hopping [10]. The system automatically estimates the distance between sensor nodes by measuring the RSSI (received signal strength indicator) at an appropriate number of sensor nodes in a localization system based on Zigbee standard. Amount of data should be collected and controlled by changing the RSSI threshold for position estimation accuracy [11].

\section{PROBLEM DISCRIPTION}

As described in the introduction section the main problem with the Zigbee network is the traffic load, as the traffic load increase the performance of the network isdecreased, thus required investigation of performance under the continuous increasing traffic load on network and their effects over their performance. Moreover it required a newframework to increase the throughput of the Zigbee cluster tree network. To complete our target we include the following work:

\subsection{Proposed Work}

A Zigbee is a low-cost communication network, which allow us to send data wireless connectivity in applications with limited power and a low cost because it is a personal wirelessnetwork and In order to achieve higher performance a cluster tree concept is used .In a cluster type topology all the nodes having a tree like structure .ZigBee cluster-tree topology may not be able to provide sufficient bandwidth for the increased traffic load, the additional information may not be delivered successfully. The proposed framework provides more flexible routing in the adaptive parent framework.

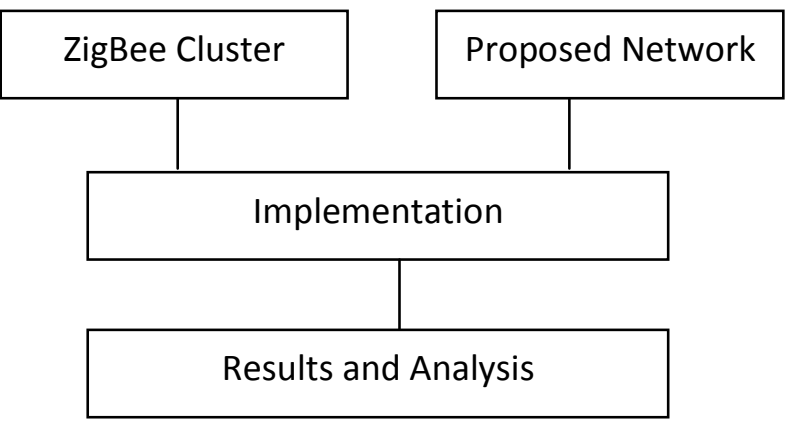

Fig.3Implementation plan

There are three types are devices in a Zigbeenetwork

1.) Pan coordinator

2.) Zigbee router

3.) End devices .

In a network all the responsibility of the communication is on the PAN coordinator, which can start a communication in that network by sending a beacon message, it provides an initialization, maintenance and control over the network the router can forward that to the destination or source. They may be work like source or sink, so we make a framework that allows coordinator and router to distribute their load on their neighbor devices, which is not in any communication link. Due to these the load is distributes and our source and sink can communicate much more flexible way . method.

There are some steps for designing proposed

1. Implement traditional ZIGBEE network and evaluate the performance parameters under load change policies.

2. Propose a new network to optimize the throughput of the system.

3. Evaluate their performance and compare with previous results.

Time duplex method applying to the solution for the above problem. Here the combination of super frame structure and an adoptive-parent-basedframework apply on ZigBee cluster-tree network. Hence there is a requirement to developed a new framework which shows better suitable result for above problem and then analysis the result with previous Zigbee cluster tree. 


\section{EXPERIMENT ANALYSIS}

Demonstration experiment for proposed method is performed via simulation for new framework. The topology of the cluster under the investigation based on Zigbee standard. In the experimental setup based taken as described in [2] and [5], and consideration according to that increasing traffic load not provided bandwidth utilization in the network. Firstly, Algorithm is applied on 10 nodes then after 20 simultaneously, increase the network through nodes till 70 nodes.The algorithm introduce only a minor overhead in terms of network density. The overhead is due to only traffic load in the network then throughput is not optimal as well a pdr (packet delivery ratio) and energy efficient in network. Through the simulation NS-2.35 we are increasing the network size simultaneously with maintaining distance between nodes also called as density. And comparing that old WPAN to the new WPAN in terms of throughput, pdr and also comparing energy efficiency in networks.

\subsection{Packet Delivery Ratio}

The packet delivery ratio (PDR) between new -WPAN and old-WPAN in terms of percentages. The packet delivery ratio is the ratio of between received packet and send packets but they both values should be from same layer i.e. all from application layer or network layer, etc.

$P D R$

$=\frac{\text { totalsuccessfullydevliverdpacket } * 100}{\text { totalsendpackets }}$

Packet delivery ratio also defined as successfully delivered packets from the overall send packets. According to the evaluated data we compare the difference in packet delivery in the networks.

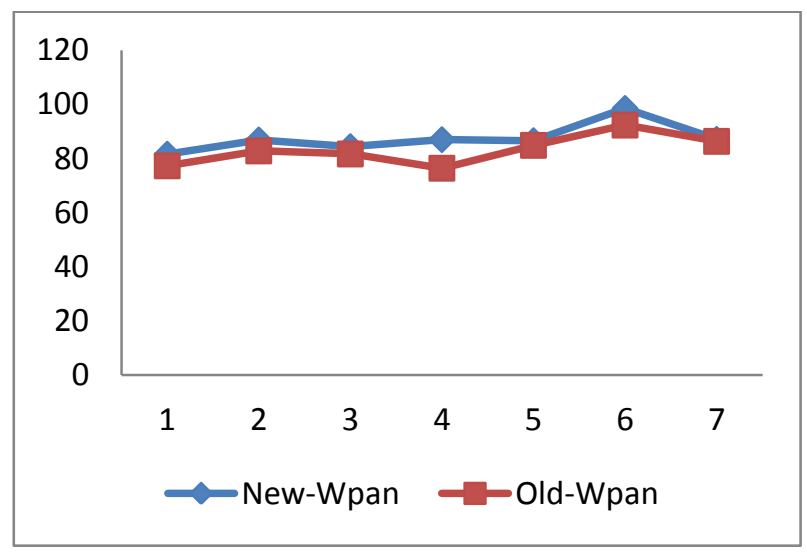

Fig.5 Packet Deliver Ratio in \%
New-WPAN gives better delivery than the oldWPAN. At each no. of nodes we can analyses the different delivery ratio between then. Evaluated data given in table 2 .

\begin{tabular}{|l|l|l|}
\hline No of Nodes & New-WPAN & Old-WPAN \\
\hline 10 & 81.6013856 & 77.2845031 \\
\hline 20 & 86.8625277 & 82.7931034 \\
\hline 30 & 84.3683084 & 81.6663096 \\
\hline 40 & 86.9632116 & 76.4111705 \\
\hline 50 & 86.5842574 & 84.8571429 \\
\hline 60 & 98.5061511 & 92.3417085 \\
\hline 70 & 87.0829361 & 86.3417085 \\
\hline
\end{tabular}

\subsection{Throughput}

Fig .4 shows the throughput between new throughput and old throughput. Throughput is calculated at each increasing node. According to data transferring through a network.

\begin{tabular}{|l|l|l|}
\hline $\begin{array}{l}\text { Number of } \\
\text { nodes }\end{array}$ & New-Throughput & Old-Throughput \\
\hline 10 & 1217.24029 & 1160.9402 \\
\hline 20 & 1250.78451 & 1230.35961 \\
\hline 30 & 1209.87495 & 1160.15765 \\
\hline 40 & 1207.05327 & 1198.23458 \\
\hline 50 & 1205.87163 & 1201.98674 \\
\hline 60 & 1109.35965 & 987.265473 \\
\hline 70 & 1250.12855 & 1198.95754 \\
\hline
\end{tabular}

Table. 1 Throughput in Networks

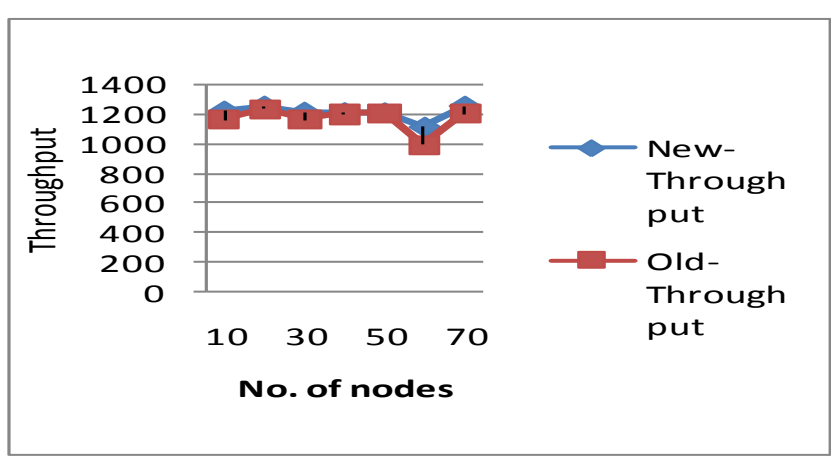

\subsection{Energy Efficient}

Energy is also a part of sensor network its lifetime the network. It's our requirements saving the energy of each node as long as possible in the network. So, an energy efficient network from source to destination is required. 
Through proposed method, get result forenergy in the source node to destination nodes. Table 3 givesthe results of the

\begin{tabular}{|l|l|l|l|l|}
\hline $\begin{array}{l}\text { No Of } \\
\text { Nodes }\end{array}$ & $\begin{array}{l}\text { Source- } \\
\text { node }\end{array}$ & Destination & Old-Source & $\begin{array}{l}\text { Old- } \\
\text { Destination }\end{array}$ \\
\hline 10 & 3.360605 & 2.993609 & 0.000061 & 0.349619 \\
\hline 20 & 6.9808 & 6.947454 & 9.311337 & 9.311337 \\
\hline 30 & 5.326064 & 4.295102 & 7.610184 & 6.980269 \\
\hline 40 & 4.450963 & 5.005272 & 0.000127 & 0.325544 \\
\hline 50 & 6.037444 & 5.992853 & 7.762074 & 7.352073 \\
\hline 60 & 7.400181 & 8.550717 & 9.170841 & 9.255389 \\
\hline 70 & 5.625607 & 5.503982 & 9.170841 & 9.255389 \\
\hline
\end{tabular}

Table 3 Energy Efficiency

experimental and graphical represent by Fig. 6 shows the energy in joules.

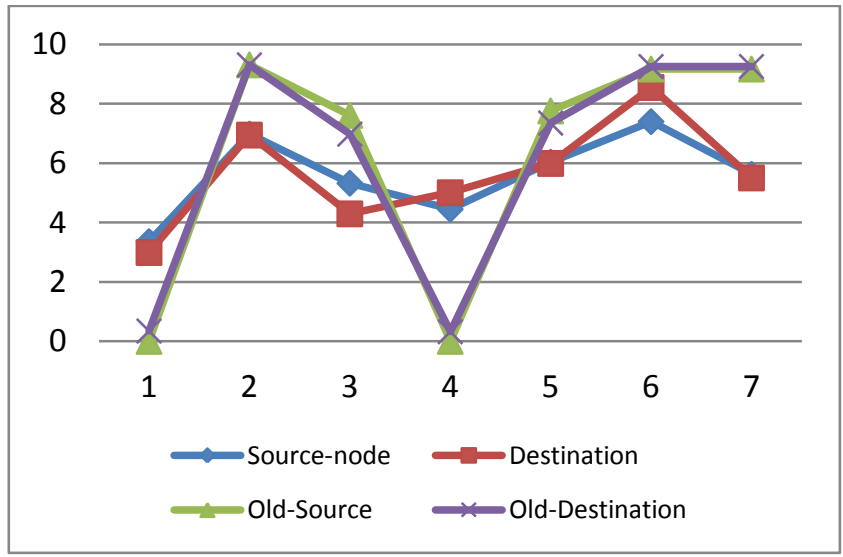

Fig. 6 Energy in Joules from Source to Destination

\section{CONCLUSION}

Due increasing network load the network performance of the network is degraded continuously. For improving efficiency of network a new approach of network arrangement is proposed and implemented that is also known as a cluster tree organization. For simulating the performance of the system we create two different scenarios first dense and the second less dense and evaluate the performance for both. After implementation of desired cluster tree network, we conclude that the network is not much affected due to increasing the number of nodes in the network. Actual performance degradation is found when the density of the network is increasing. According to the results,be the newly arranged network perform well than old network.

\section{ACKNOWLEDGMENTS}

The authors would like to thank the anonymous reviewers for their detailed, valuable comments and constructive suggestions.

\section{REFERENCES}

[1] I.F. Akyildiz, Y. Sankarasubramaniam, W.Su, and E. Cayirci, "A Survey on Sensor Networks," IEEE Comm. Magazine, vol. 40, no. 8, pp. 102-114, Aug. 2002.

[2] Huang.Y.K.Pang.A.C, Hsiu.P.C, Zhuang.W, Liu.P,"Distributed Throughput Optimization for ZigBee Cluster-Tree Networks, "IEEE Transactions On Parallel And Distributed Systems, Vol. 23, and No. 3, March 2012

[3] Zigbee: "Wireless Control That Simply Works"William C. Craig Program Manager Wireless Communications ZMD America, Inc.

[4] Blumenthal.J, Grossmann.R, Golatowski.F, Timmermann.D,"Weighted Centroid Localization in Zigbee-based Sensor Networks,”1-4244-0830IEEE 2007

[5] Koubaa.A, Cunha.A,Alves.M,“A Time Division Beacon Scheduling Mechanism for IEEE 802.15.4/Zigbee Cluster-Tree Wireless Sensor Networks," Research Group, Polytechnic Institute of Porto, RuaAntónio Bernardino de Almeida, 431, 4200-072 Porto, Portugal

[6] A. Bhatia and P. Kaushik, "A Cluster Based Minimum BatteryCost AODV Routing Using Multipath Route forZigBee," Proc.IEEE Int'l Conf. Networks (ICON), Dec. 2008.

[7] R. Burda and C. Wietfeld, "A Distributed and Autonomous Beacon Scheduling Algorithm for IEEE 802.15.4/ZigBee Networks,"Proc. IEEE Int'l Conf. MobileAdhoc and Sensor Systems (MASS), Oct. 2007.

[8] Kim. T, Kim.D, Park.N, Yoo. S, Lopez. T.S, “Shortcut Tree Routing in ZigBee Networks" Information and Communications University, Electronics and Telecommunications Research Institute*IEEE 2007

[9] Lee. J.S, SU. Y.W,Shen.C.C,“A Comparative Study of Wireless Protocols: Bluetooth, UWB, ZigBee, and Wi$\mathrm{Fi}$," the 33rd Annual Conference of the IEEE Industrial Electronics Society (IECON) Nov. 5-8, 2007, Taipei, Taiwan

[10] Pekhterev.G,Sahinoglu. Z, Orlik. P,Bhatti.G,“Image Transmission over IEEE 802.15.4 andZigBee Networks"IEEE ISCAS May 2005

[11] IndoorLocalizationSystemUsingRSSIMeasurmentOfWir elessSensorNetworkBasedOnZigbeeStandard,Masashi Sugano, School of Comprehensive rehabilitation Osaka Prefecture University 